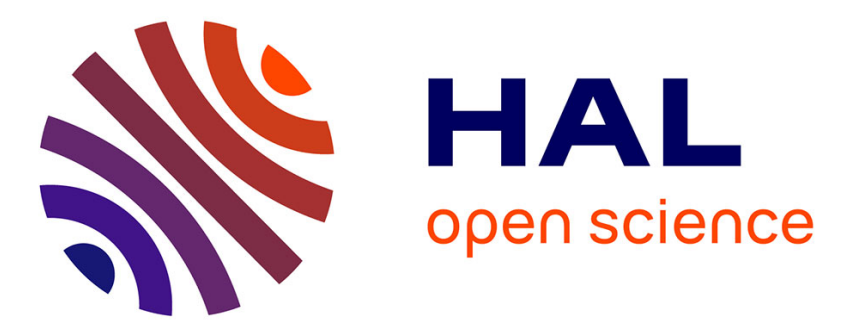

\title{
Advances in Monitoring Soil Nutrients by Near Infrared Spectroscopy
}

Yan Wang, Bei Cui, Yanhua Zhou, Xiudong Sun

\section{To cite this version:}

Yan Wang, Bei Cui, Yanhua Zhou, Xiudong Sun. Advances in Monitoring Soil Nutrients by Near Infrared Spectroscopy. 11th International Conference on Computer and Computing Technologies in Agriculture (CCTA), Aug 2017, Jilin, China. pp.94-99, 10.1007/978-3-030-06179-1_10 . hal02111526

\section{HAL Id: hal-02111526 \\ https://hal.inria.fr/hal-02111526}

Submitted on 26 Apr 2019

HAL is a multi-disciplinary open access archive for the deposit and dissemination of scientific research documents, whether they are published or not. The documents may come from teaching and research institutions in France or abroad, or from public or private research centers.
L'archive ouverte pluridisciplinaire HAL, est destinée au dépôt et à la diffusion de documents scientifiques de niveau recherche, publiés ou non, émanant des établissements d'enseignement et de recherche français ou étrangers, des laboratoires publics ou privés. 


\title{
Advances in monitoring soil nutrients by near
}

\author{
infrared spectroscopy \\ Yan Wang ${ }^{1(\bowtie)}$, Bei Cui ${ }^{2}$, Yanhua Zhou ${ }^{1}$, Xiudong Sun ${ }^{1}$ \\ ${ }^{1}$ Shanghai Vocational College of Agriculture and Forestry, Shanghai, China \\ 14432 eshafc.edu. cn \\ ${ }^{2}$ Institute of Remote Sensing and Digital Earth Chinese Academy of Sciences, Sanya, China \\ cuibeieradi.ac. cn
}

\begin{abstract}
Soil nutrients play an important role in crop growth, and traditional monitoring methods are still the first choice for high precision measurement. However, it is necessary to have a quicker and simpler way to improve the efficiency of soil nutrient monitoring because of its long monitoring time, pollution and high labor cost. Near infrared spectroscopy has become the focus of its research because of its rapid and pollution-free advantages. At present, using near infrared spectroscopy to distinguish soil types, soil heavy metal pollution technology has become increasingly mature, but the soil nutrients, such as monitoring of soil organic matter, available phosphorus, available potassium, available nitrogen is still in the research stage. This paper reviews the recent research results of soil nutrients in near infrared spectrum monitoring technology based on collation, summary and key technology of common data processing method, and analysis the advantages and disadvantages of different detection methods for soil nutrient direction of near infrared spectroscopy technology put forward suggestions to further research.
\end{abstract}

Keywords: Near infrared spectroscopy, Soil nutrients,

\section{Introduction}

Soil nutrients mainly refer to the contents of organic matter, available phosphorus, available potassium and available nitrogen in soil. Soil nutrients can provide nutrients needed for crop growth, but also constitute an important component of soil structure and determine soil physical and chemical properties. It is important for crop growth. Soil nutrient content directly influences crop yield, and it is also an important index to guide fertilization decision-making.

Traditional soil nutrient determination was completed in the laboratory by chemical reagents. Poor timeliness, high labor costs, easy to cause pollution. How to rapidly and efficiently determine soil nutrients is an urgent problem to be solved in the development of precision agriculture (precision agriculture, PA). Near infrared spectroscopy (Near Infrared Spectrometry, NIR) is an important component of 
remote sensing technology, and its advantages of nondestructive and rapid detection have become the focus of research. Different soil texture, water storage capacity and soil particle size make it possible to detect soil nutrient content by near infrared spectroscopy. At present, the technology of near infrared spectroscopy to distinguish soil types and soil heavy metals pollution is becoming more and more mature. But the application of some soil nutrients content is not yet mature. In this paper, the application of near infrared spectroscopy in soil nutrient testing was analyzed, in order to promote the application of NIR in soil nutrient testing and improve the speed and efficiency of PA development.

\section{Recent research status of in monitoring soil nutrients by near infrared spectroscopy}

Soil indicators closely related to crop growth include organic matter, available phosphorus, available potassium and available nitrogen. The detection of soil nutrient indexes will help to understand the overall situation of soil, guide the rational allocation of land resources in the field, and make fertilization decisions and predict the quality and output of agricultural products. The research of rapid and efficient soil nutrient detection technology is the focus of recent research. NIR has become the research focus of soil nutrient detection technology because of its nondestructive, rapid and other advantages.

Soil organic matter refers to organic matter containing carbon in soil, including residues of various animals and plants, microorganisms and organic matter [1], which are decomposed and synthesized. Soil organic matter plays an important role in soil formation and soil fertility. The contents of available phosphorus, available potassium and available nitrogen in soil reflect the storage and supply ability of nutrients in soil to a certain extent.

The study of soil organic matter content by NIR began in the middle of the 1980s. In recent years, there are many achievements, and the soil organic matter sensitive bands, detection methods and models are also gradually deepened. The research of near infrared spectroscopy in China started late. In recent years, many scholars have made great efforts to explore the methods of detecting soil nutrients by near infrared spectroscopy.

Gao Hongzhi et al. (2011) using FOSSXDS Near Infrared Spectroscopy Analyzer in 85 soil samples collected from northeast spectra, the correlation coefficient spectrum and successive projection algorithm for near infrared spectral analysis of the soil, and excellent characteristics of wavelength of total nitrogen and organic matter were selected. A high signal-to-noise ratio near infrared spectroscopy system based on the characteristic wavelength was developed, and the near infrared spectra and measurement results of soil samples were analyzed. The system can effectively measure total nitrogen and organic matter content in soil nutrients.

Zhang Juanjuan et al. (2012) the 5 main types of soil in the research China in central and eastern regions as the research object, various pretreatment methods combined 
treatment, combined with partial least squares (PLS) calibration model for each spectral region. The results show that the near infrared spectrum can be used to estimate soil total nitrogen, and a better prediction result can be obtained by using the frequency band $(4000 \sim 5500 \mathrm{~cm}-1)$ as the modeling area.

Li Jie et al. (2012) in 72 soil samples collected from the Beijing suburb of an experimental field as test materials, the application of $\mathrm{Fu}$ Liye transform near infrared spectroscopy analysis of total nitrogen, soil total potassium, organic matter, nutrient content and $\mathrm{pH}$ value. By using partial least squares (PLS) on the measured data of soil nutrient and spectral values using regression analysis to establish prediction model, the results show that the established method of partial least squares regression model can accurately predict the nutrient in Beijing cinnamon soil soil total nitrogen and organic matter, total potassium and $\mathrm{pH}$ value of 4 was predicted based on nutrient.

Song Haiyan et al. (2012) by orthogonal signal correction (OSC) method can be correlated with the concentration profiles of the advantage of picture information, the corrected information mapping and partial least squares (PLS) method combined by near infrared spectroscopy of different soil texture, soil, clay loam sand: discriminant analysis.

Liu Xuemei et al. (2013) using visible / short wave near infrared spectroscopy (Vis/SW-NIR) analysis to measure soil available nitrogen $(\mathrm{N})$ and available potassium $(\mathrm{K})$. The application of genetic algorithm in soil nutrient analysis is discussed. The correction model is established by least squares support vector machine (LS-SVM) according to the optimization results. The results show that the visible / short wave near infrared spectrum based on the genetic algorithm can be used as a method for the determination of soil physical and chemical properties by using LS-SVM modeling.

Wang Chang et al. (2013) in the middle and lower reaches of Yangtze River main grain producing area of paddy soil as the research object, collecting 17 kinds of different treatments of 136 soil samples in $350 \sim 2500 \mathrm{~nm}$ near infrared spectroscopy, using partial least squares regression analysis the quantitative analysis model of total carbon, total nitrogen, carbon and nitrogen combined with cross validation method of near infrared diffuse a method of analysis of reflectance spectra measured with the traditional chemical ratio, available potassium, available phosphorus, soil conductivity, soil $\mathrm{pH}$ index. The results show that the total carbon, total nitrogen, carbon to nitrogen ratio and $\mathrm{pH}$ model are very good. The predicted results of the available $\mathrm{K}$ model are good, while the results of the available $\mathrm{P}$ and conductivity models are very unsatisfactory.

Wu Qian et al. (2014) to study the paddy soil in Yuxi tobacco science and technology demonstration park in Yunnan Province, 6 nitrogen levels, 144 soil samples, the spectral modeling method of the content of soil available nitrogen, phosphorus and potassium in the fast estimation, by far scatter correction and first derivative spectral preprocessing, through spectral correlation analysis of feature 
selection and application of local bands, nonlinear regression analysis modeling method, results show that using the local BP neural local modeling method to establish the soil available nitrogen, phosphorus and potassium content of the quantitative analysis model, which can realize the rapid diagnosis of soil nutrients. Fang Xiaorong et al. (2015) based on near infrared spectroscopy combined with continuous projection algorithm and regression coefficient analysis, the total nitrogen content of soil was studied. The near infrared spectrum data of farmland soil samples were collected, and the total number of soil samples was 394. Partial least squares regression (PLS), multiple linear regression (MLR) and least squares support vector machine (LS-SVM) modeling were adopted to establish the prediction model of total nitrogen. The results show that the characteristic wavelength based on the continuous projection algorithm and the regression coefficient analysis can be applied to the detection of total nitrogen in soil by near infrared spectroscopy. At the same time, the method used in this study can simplify the model and be suitable for developing portable soil nutrient detector. Zeng Jing et al. (2015) were studied in purple soil of Sichuan Chongqing area, analysis of soil moisture influence on prediction accuracy of soil organic matter content in purple, and the conversion of near infrared spectral absorbance of certain water content under dry soil conditions was then to establish the prediction model of near infrared spectroscopy of organic matter content by PLS method. Compared with original model prediction accuracy has improved significantly.

He Dongjian et al. (2015) acquisition experiment field of Northwest Agriculture and Forestry University and the surrounding farmland soil total 160, synchronous acquisition of soil available phosphorus content and the corresponding near infrared spectral data, respectively, using four kinds of modeling methods, finally found modeling method of least squares support vector machine method can effectively predict the content of soil available phosphorus.

Wu Jinzhuo et al. (2016) to explore the method of near infrared spectroscopy in field rapid determination of soil total nitrogen and available nitrogen content, soil spectral signal acquisition, combined with partial least squares method and principal component analysis method, respectively. The calibration model for determination of nitrogen content of soil total nitrogen and alkali solution is established.

Table 1. Evaluation indices of the calibration models of soil TN and AN by two pretreatment methods combined with PLS modeling approach

\begin{tabular}{|c|c|c|c|c|}
\hline $\begin{array}{l}\text { Modeling } \\
\text { object }\end{array}$ & Model & Spectral preprocessing method & $\mathrm{R} 2$ & RMSE \\
\hline \multirow[t]{4}{*}{$\mathrm{TN}$} & Calibration set & Multivariate scattering correction & 0.7549 & 0.1829 \\
\hline & & Wavelet de-noising & 0.8385 & 0.1521 \\
\hline & Validation set & Multivariate scattering correction & 0.7339 & 0.1895 \\
\hline & & Wavelet de-noising & 0.7549 & 0.1842 \\
\hline $\mathrm{AN}$ & Calibration set & Multivariate scattering correction & 0.7879 & 0.2018 \\
\hline
\end{tabular}




\begin{tabular}{llll} 
& Wavelet de-noising & 0.8665 & 0.0077 \\
Validation set & Multivariate scattering correction & 0.7113 & 0.2308 \\
& Wavelet de-noising & 0.7961 & 0.0094 \\
\hline
\end{tabular}

Table 1 shows the spectral data after wavelet de-noising based on near infrared spectroscopy combined with PLS method to establish the model of soil total nitrogen and available nitrogen content, the correlation coefficient of the calibration set and validation set were higher than that of near infrared spectra by the multiple scattering correction, RMES is on the contrary, show that wavelet transform the de-noising effect is better than the multiple scattering correction de-noising effect.

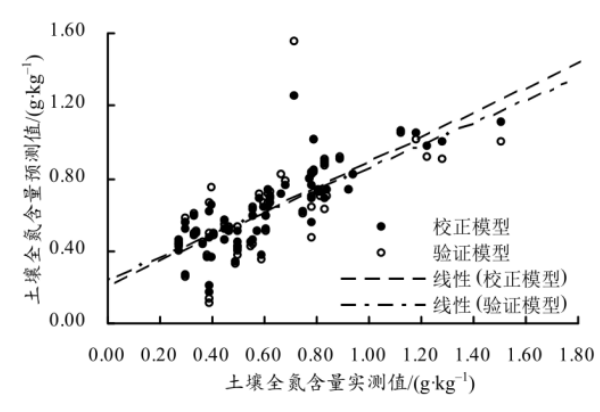

Fig.1. Relationship between

predicted and measured values

of soil total nitrogen

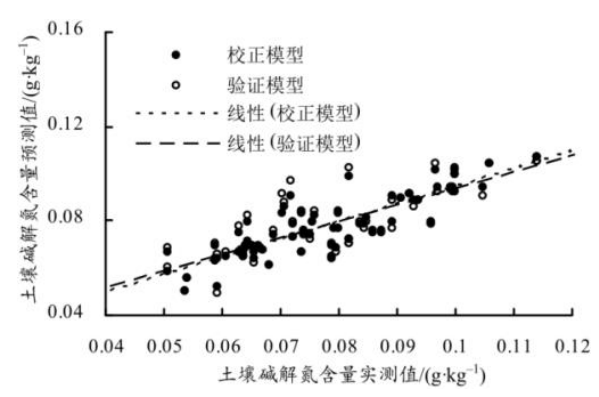

Fig.2. Relationship between predicted and measured values of soil available nitrogen

Relationships between predicted and measured values of soil total nitrogen and available nitrogen are shown in fig. 1 and fig.2. Comprehensive analysis results show that the application of near infrared spectroscopic techniques on soil total nitrogen and available nitrogen content of quantitative prediction is feasible, and the application of wavelet transform to preprocess spectral redundancy, and partial least squares method can effectively improve the accuracy of the model is significantly improved.

\section{Conclusion}

\subsection{The methods of data analysis in different regions still need to be explored}

The research on soil nutrients detection by NIR mainly focuses on the data analysis method. Data analysis mainly refers to the pretreatment mode and modeling method of near infrared spectrum data, and the purpose of modeling is to explore the stable conventional model for predicting soil nutrient content. Most of the existing researches are based on specific regions, and data analysis methods suitable for monitoring soil nutrients in various regions are still being explored. Future research on universal data analysis method is still the key direction for research and development of soil nutrient detection.

\subsection{The gap processing of spectral data between different years still needs to be explored}

The pretreatment of spectral data is mainly aimed at eliminating the influence of 
water, particle size and soil type on soil nutrient detection, and improving the universality and stability of the model. At present, the research results show that the spectral data preprocessing way absorbance, mean normalization, 5 point mean filter smoothing processing can effectively increase the prediction accuracy of the model. But the spectral data are mostly data at the same time, and there are significant differences in the measured spectral data in different years. In the future, narrowing the gap between spectral data years is still a direction of research.

\section{3 the general modeling method between different years still needs to be explored}

The modeling method has been greatly improved. From the early linear regression analysis to the current partial least squares, local neural network analysis and least squares support vector machine, the prediction accuracy of the model has been significantly improved. But because of the soil type and the time of soil testing, the prediction effect of the model in different years is decreasing year by year. In order to develop a rapid detection instrument for Soil Nutrients Based on near infrared spectroscopy, the stability and universality of the model are still an important direction of the research.

\section{References}

1. DOU Sen. Soil organic matter[M]. Beijing: Science Press. (in Chinese) (2010)

2. Gao Hongzhi, Lu Qipeng.: Near infrared spectroscopy analysis and measuring system for primary nutrient of Soil, J. Spectroscopy and Spectral Analysis. 31(5): 1245-1249(in Chinese with English abstract )(2011)

3. Zhang Juanjuan, Tian Yongchao, Yao Xia, et al.: Estimating model of soil total nitrogen content based on near-infrared spectroscopy analysis, J. Transactions of the Chinese Society of Agricultural Engineering (Transactions of the CSAE). 28(12): 183 - 188(in Chinese with English abstract)(2012)

4. He Dongjian, Yang Chenghai, Yang Qing, et al.: Research progress of real-time measurement of soil attributes for precision agriculture, J. Transactions of the Chinese Society of Agricultural Engineering (Transactions of the CSAE). 28(12): 183 - 188(in Chinese with English abstract)(2012)

5. Song Haiyan.Qin Gang,Han Xiaoping,et al.: Soil classification based on near infrared reflectance spectroscopy and orthogonal signal correction-partial least square, J. Transactions of the Chinese Society of Agricultural Engineering (Transactions of the CSAE). 28(7): 168 - 171(in Chinese with English abstract)(2012)

6. Li Jie, Zhang Xiaochao, Yuan Yanwei, et al.: Analysis of soil nutrient content based on near infrared reflectance spectroscopy in Beijing region, J. Transactions of the CSAE. 28(2):176 - 179(in Chinese with English abstract)( 2012)

7. Liu Xuemei, Zhang Hai Liang.: Study on Soil Nutrients by Using Near-Infrared Spectroscopy Based on the GA method, J. Journal of Irrigation and Drainage. 32(2):138-141 (in Chinese with English abstract)(2013) 
8. Wang chang, Huang Chichao, Yu Guanghui, et al.: Rapid evaluation of soil quality through a near infrared-partial least squares (NIR-PLS) method, J. Acta Pedologica Sinica. 50(5):881-890 (in Chinese with English abstract) (2013)

9. Wu Qian, Yang Yuhong, Xu Zhaoli, et al.: Applying local neural networks and visible / Near Infrared Spectroscopy to estimating available nitrogen, phosphorus and potassium in soil, J. Spectroscopy and Spectral Analysis. 34(8):2102-2105(in Chinese with English abstract )(2014)

10. ZENG jing, Li Guanglin, Zhou Shengling.: A Research of the Effects of Soil Moisture on NIR Spectroscopic Detection of Organic Matter Content in Purple Soil, J. Journal of Southwest University (Natural Science Edition). 37(10):167-173(in Chinese with English abstract )(2015)

11. Fang Xiaorong, Huang Lingxia, et al.: spectroscopy and spectral detection Measurement of soil total nitrogen using near infrared spectroscopy combined with RCA and SPA, J. Spectroscopy and Spectral Analysis. 35(5):1248-1252 (in Chinese with English abstract )(2015)

12. Wu Jinzhuo, Kong Linlin, Li Ying, et al.: Prediction models of total and available soil nitrogen based on near infrared spectroscopy, J. Journal of Hunan Agricultural University (Natural Sciences). 42 (1) :91-96(in Chinese with English abstract )(2016) 